\title{
Operação de Glenn bidirecional
}

\author{
Paulo CHACCUR*, Jarbas J. DINKAUYZEN*, Camilo ABDULMASSIH NETO*, Antoninho S. ARNONI*, \\ Maria Virgínia D. SILVA*, leda Jatene BOSíSIO*, Valmir F. FONTES*, Márcia CAPPELLARI*, Paulo P. \\ PAULISTA*, Adib D. JATENE*, Luiz Carlos Bento de SOUZA*
}

RBCCV 44205-179

CHACCUR, P.; DINKHUYSEN, J. J.; ABDULMASSIH NETO, C.; ARNONI, A. S.; SILVA,M. V. D.; BOSÍSIO, I. J.; FONTES, V. F.; CAPPELLARI, M.; PAULISTA, P. P.; JATENE, A. D.; SOUZA, L. C. B. - Operaçāo de Glenn bidirecional. Rev. Bras. Cir. Cardiovasc., 7(3):194-200, 1992.

RESUMO: A cirurgia de Gleen bidirecional tem sido empregada como uma opção ao tratamento cirúrgico de pacientes considerados candidatos "não ideais" à cirurgia de derivação átrio-pulmonar. A operação consiste na anastomose da veia cava superior com a artéria pulmonar (anastomose término-lateral), permitindo o fluxo sangüíneo também para o pulmāo contra-lateral. A partir de janeiro de 1990 até fevereiro de 1992, 20 pacientes foram operados em nosso Serviço, com idade variando de 5 meses a 8 anos (média de 37,7 meses). Oito pacientes eram do sexo feminino e o peso variou de 6,3 a $18,8 \mathrm{Kg}$ (média $-12,4 \mathrm{~kg}$ ). A indicação cirúrgica foi considerada primária em 10 casos. Sete casos de atresia tricúspide e 3 de ventrículo único, que apresentavam acentuada diminuiçāo do fluxo pulmonar e não eram candidatos a correçāo total funcional. Os demais pacientes tiveram a indicação considerada secundária, ou seja, já haviam sido submetidos a operação de shunt artério-venoso, cerclagem do tranco pulmonar, ou atriosseptostomia, 8 casos de atresia tricúspide, 3 de ventrículo único com estenose ou cerclagem pulmonar e 1 caso de DVSVD com ventrículo superior-inferior). A operaçāo foi realizada com desvio da veia cava-átrio direito em 12 casos e como uso de CEC em 8. Durante o procedimento cirúrgico, os pacientes foram monitorizados com oxímetro pulsátil e a saturaçāo de oxigênio média pré-correçāo foi de $75,5 \%$ (71\% a 86\%) e após, de $95 \%$ (91\% a $98 \%)$. Não ocorreu óbito hospitalar e o único óbito tardio foi devido a infecçāo pulmonar no $2^{\circ}$ mês de pós-operatório. Achamos, portanto, que a operaçăo de Glenn bidirecional estará bem indicada como primeira etapa da correçāo definitiva, pois não aumenta o trabalho cardíaco e a resistência vascular pulmonar não produz distorçōes em artéria pulmonar como shunt tipo Blalock-Taussig.

DESCRITORES: Glenn bidirecional, cirurgia de.

\section{INTRODUÇĀO}

A anastomose cavo-pulmonar ( $A C P$ ) pela técnica de GLENN ${ }^{10}$ foi pela primeira vez utilizada clinicamente em 1958, em um menino de 7 anos de idade portador de ventrículo único com estenose pulmonar. Alguns anos antes, na Itália, CARLTON et alii ${ }^{5}$ estudaram experimentalmente este tipo de anastomose, tendo sido divulgada internacionalmente alguns anos após. Esta técnica consistia na secção transversa da artéria pulmonar direita com sutura de sua porção proximal e anastomose da porção distal lateralmente na cava superior ao nível da desembocadura da veia ázigos, que havia sido ligada e seccionada, e, final- mente, efetuada a ligadura da cava superior próximo à junçāo com o átrio direito (Figura 1).

Com persistência exemplar, Dr. Glenn continuou os estudos experimentais de desvio parcial do coração direito por mais 10 anos, tendo operado aproximadamente 100 cães ${ }^{4,11,20,21}$. A partir de então, descreveu a técnica da anastomose direta términoterminal cavo-pulmonar, permanecendo padronizada por vários anos (Figura 2).

Durante esse período, os cirurgiōes tentaram o desvio parcial ou total do coraçāo direito. SHUMACKER ${ }^{23}$ referiu esta técnica experimentalmente, em 1954, e

Trabalho realizado no Instituto Dante Pazzanese de Cardiologia. São Paulo, SP, Brasil.

Apresentado ao $19^{\circ}$ Congresso Nacional de Cirurgia Cardíaca. São Paulo, 7 a 9 de maio, 1992.

* Do Instituto Dante Pazzanese de Cardiologia.

Endereço para separatas: Paulo Chaccur. Av. Dr. Dante Pazzanese, 500. Ibirapuera. 04531 Sảo Paulo, SP, Brasil. 
CHACCUR, P.; DINKHUYSEN, J. J.; ABDULMASSIH NETO, C.; ARNONI, A. S.; SILVA,M. V. D.; BOSÍSIO, I. J.; FONTES, V. F.; CAPPELLARI, M.; PAULISTA, P. P.; JATENE, A. D.; SOUZA, L. C. B. - Operaçäo de Glenn bidirecional. Rev. Bras. Cir.
Cardiovasc., 7(3):194-200, 1992.
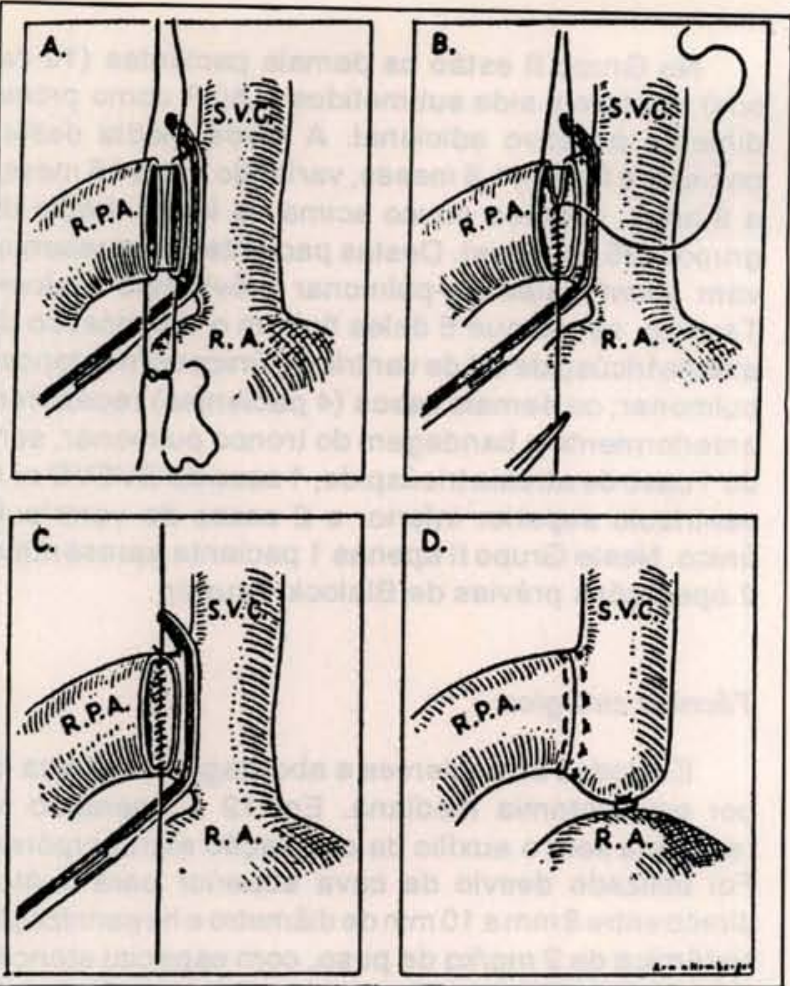

Fig. 1 - Técnica de anastomose da veia cava superior com a artéria pulmonar direita ( $R A=$ átrio direito; $R P A=$ artéria pulmonar direita: SVC = veia cava superior. (New England Journal of Medicine 1958).

mencionou 2 insucessos clínicos para esse tipo de anastomose. Em 1956, ROBCSEK et alii ${ }^{22}$ relataram sua experiência em 15 căes, apresentando os primeiros casos de sucesso com 6 meses de pós-operatório; o seu primeiro caso clínico foi operado em 1959, pouco tempo após a publicação de Glenn, e continuou trabalhando nos 12 anos seguintes, tanto experimental como clinicamente.

A experiência com ACP na Uniāo Soviética, além dos trabalhos de Robicsek, foi realizada por BAKULJEV \& KELESNIKOV ${ }^{3}$. Em 1956, MESHALKIN ${ }^{19}$ relatou 24 casos de crianças tratadas com ACP com 21 sobrevidas, tendo sido o primeiro relato clínico com sucesso.

Modificaçōes técnicas na anastomose entre a veia cava superior com a artéria pulmonar direita foram realizadas porvários grupos, com direcionamento do sangue da cava superior para os pulmōes direito e esquerdo, através da anastomose término-lateral cavo-pulmonar. Desde 1967, ABRAMS $^{2}$ tem realizado essas anastomoses em 38 pacientes com baixa mortalidade, mas os seus resultados só foram divulgados em 1988. Em 1966, HALLER et alii ${ }^{12}$ realizaram experimentalmente a ACP sem ligar proximalmente a artéria pulmonar direita e, assim, obteve a anastomose cavo-pulmonar bi-direcional (ACP-bi).
Mais recentemente, em 1984, KAWASHIMA et alii ${ }^{13}$, utilizaram a ACP-bi de forma paliativa com sucesso e DE LEVAL et alii ${ }^{7}$ demonstraram as possíveis vantagens da $\mathrm{ACP}$ total, associando à anastomose de Glenn bi-direcional a tunelizaçāo da cava inferior para a cava superior, anastomosando-a na borda inferior da artéria pulmonar direita. Até 1989, poucas informaçōes estavam avaliáveis quanto ao uso dessa anastomose como primeiro estágio para posterior correçāo definitiva, ou como operação paliativa definitiva. Alguns resultados foram publicados por LAMBERTI et alii ${ }^{17} \mathrm{e}$ por MAZZERA et alii ${ }^{18}$. Esses autores concluíram que, em alguns casos considerados năo ideais para cirurgia de Fontan, mas que poderão ser no futuro, a ACP-bi poderá ser considerada como primeiro estágio para a cirurgia paliativa definitiva. Entretanto, se, por várias razōes, a cirurgia de Fontan for considerada de alto risco, ou se houver insucesso durante a correçāo tipo Fontan, a ACP poderá ser considerada uma paliação definitiva.

O presente trabalho relata nossa experiência com ACP-bi em 20 crianças portadoras de cardiopatias congênitas cianogênicas complexas.

\section{CASUÍSTICA E MÉTODOS}

A primeira operação de Glenn bidirecional, em nosso Serviço, foi a realizada em setembro de 1990 , em uma menina de 5 anos e 7 meses de idade portadora de ventrículo único tipo direito, atresia mitral, veia cava superior esquerda persistente e bandagem do tronco pulmonar, que havia sido realizada em 1985. Desde entāo, até fevereiro de 1992 , outros 19 casos foram operados. A idade média dos

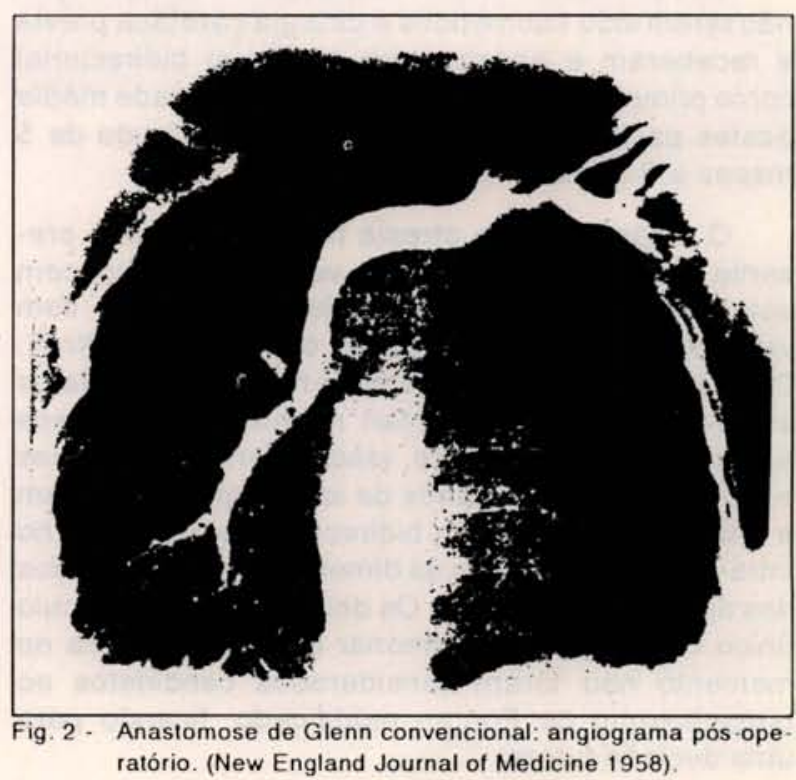


CHACCUR, P. DINKHUYSEN, J. J.; ABDULMASSIH NETO, C.; ARNONI, A. S.; SILVA,M. V. D.; BOSÍSIO, I. J.; FONTES, V. F.; CAPPELLARI, M.; PAULISTA, P. P.; JATENE, A. D.; SOUZA, L. C. B. - Operaçāo de Glenn bidirecional. Rev. Bras. Cir. Cardiovasc., $7(3): 194-200,1992$.

pacientes foi de 37,7 meses, variando entre 5 meses a 8 anos. O sexo masculino predominou com 12 $(60 \%)$ casos. O peso médio foi de $12,4 \mathrm{~kg}$, variando entre 6,3 a $18,8 \mathrm{~kg}$.

A cardiopatia congênita cianogênica mais freqüente foi a atresia tricúspide com 13 (65\%) casos, seguida em freqüência pelos casos de ventrículo único ( 6 casos) com estenose ou bandagem do tronco pulmonar e, finalmente, 1 caso de dupla via de saída do ventrículo direito com ventrículo superior e inferior, comunicação interventricular e hipoplasia da valva mitral.

Todos os pacientes apresentavam cianose de grau variável, sendo necessária hemodiluição como preparaçāo para o estudo hemodinâmico e posterior operaçāo ( 2 casos). O outro sintoma freqüente, neste grupo de pacientes, foi a dispnéia aos médios e pequenos esforços. Em 1 caso de atresia tricúspide e operaçăo de Blalock-Taussig à esquerda, os sinais de insuficiência cardíaca estiveram presentes.

Todas a crianças foram submetidas a estudo ecocardiográfico e estudo hemodinâmico para análise da pressāo diastólica final do ventrículo esquerdo, de pressōes em tronco e artérias pulmonares, e estudo contrastado para avaliação da anatomia da circulaçāo extra e intraparanquimatosa pulmonar. Um único paciente apresentava estenose na origem da artéria pulmonar esquerda que foi corrigida com amplicaçāo com pericárdio bovino. A pressão média do tronco pulmonar nāo foi medida diretamente em todos os casos devido à dificuldade técnica em se atingir a circulação pulmonar, variando entre $10 \mathrm{mmHg}$ e $20 \mathrm{mmHg}$ (média de $14 \mathrm{mmHg}$ ) e a pd2 da VE esteve entre $7 \mathrm{mmHg}$ e $15 \mathrm{mmHg}$ (média $11 \mathrm{mmHg}$ ).

Dez pacientes foram colocados no Grupo I por não terem sido submetidos a cirurgia cardíaca prévia e receberam a anastomose de Glenn bidirecional como primeiro procedimento paliativo. A idade média destes pacientes foi de 35,2 meses, variando de 5 meses a 8 anos.

O diagnóstico de atresia tricúspide estava presente em 7 pacientes e de ventrículo único com estenose pulmonar em 3 pacientes ( 2 casos com valva AV única e em 1 caso com atresia mitral). Destes 10 casos, apenas 8 pacientes são candidatos ao procedimento de Fontan modificados; 6 casos apresentam, no momento, idade inferior a 19 meses e 2 casos, com 7 e 8 anos de idade, que receberam a anastomose de Glenn bidirecional por decisão no intra-operatório, devido às dimensōes algo reduzidos das artérias pulmonares. Os dois casos de ventrículo único com estenose pulmonar e valva $A V$ única no momento năo foram considerados candidatos ao procedimento de Fontan modificado, ficando para uma decisão futura.
No Grupo II estăo os demais pacientes (10 casos) por terem sido submetidos a ACP como procedimento paliativo adicional. $\mathrm{A}$ idade média destes pacientes foi de 41,8 meses, variando entre 15 meses a 8 anos, estando pouco acima da idade média do grupo I (35,2 meses). Destas pacientes, 6 apresentavam shunt sistêmico-pulmonar prévio tipo BlalockTaussig, sendo que 5 deles tinham o diagnóstico de atresia tricúspide 1 de ventrículo único comestenose pulmonar; os demais casos (4 pacientes) receberam anteriormente a bandagem do tronco pulmonar, sendo 1 caso de atresia tricúspide, 1 caso de DVSVD com ventrículo superior inferior e 2 casos de ventrículo único. Neste Grupo II apenas 1 paciente apresentava 2 operaçōes prévias de Blalock-Taussig.

\section{Técnica cirúrgica}

Em todos os pacientes a abordagem cirúrgica foi por esternotomia mediana. Em 12 a operaçāo foi realizada sem o auxílio da circulaçāo extracorpórea. Foi utilizado desvio da cava superior para o átrio direito entre $8 \mathrm{mma} 10 \mathrm{~mm}$ de diâmetro e heparinização sistêmica de $2 \mathrm{mg} / \mathrm{kg}$ de peso, com especial atençāo para evitar embolia gasosa durante o shunt. A veia cava superior era seccionada próximo à junçāo com o átrio direito, com atenção para nāo traumatizar o nó sinusal; a porção proximal era suturada com dois planos de fio monofilamentar $5-0$ ou $6-0$ e a porçāo distal ou cefálica era anastomosada término-lateralmente na artéria pulmonar direita em sutura contínua com Prolene 7-0 ancorados a 180. Em 4 casos a presença da veia cava superior esquerda permitiu uma segunda ACP para a artéria pulmonar esquerda, utilizando-se também o shunt cava esquerda-átrio direito.

A anastomose deve ser realizada com muito cuidado, porque qualquer torçāo poderá interferir com o fluxo de drenagem venosa. Após o término da anastomose, o shunt temporário era removido e a heparina neutralizada caso houvesse sinais de sangramento. O pericárdio poderá ser aproximado e o tórax drenado de forma convencional.

Em 8 pacientes a anastomose de Glenn bidirecional foi realizada como auxílio da circulação extracorpórea. Em 3 casos a presença de shunt sistêmico-pulmonar tipo Blalock-Taussig à direita obrigou a esse procedimento. Em 4 casos a indicaçāo foi efetuada devido a dificuldade técnica e em 1 caso de ventrículo único tipo $E$, bandagem do TP e aorta em L posição que havia sido submetida a correçāo total tipo Fontan, mas que evoluiu com baixo débito importante no período pós-perfusāo, necessitando de manobra de take-down e optada pela realizaçāo da ACP-bi.

Três pacientes apresentavam idade inferior a 2 anos e 6 meses e os demais (4) foram considerados 
CHACCUR, P.; DINKHUYSEN, J. J.; ABDULMASSIH NETO, C.; ARNONI, A. S.; SILVA,M. V. D.; BOSÍSIO, I. J.; FONTES, V. F CAPPELLARI, M.; PAULISTA, P. P.; JATENE, A. D.; SOUZA, L. C. B. - Operaçäo de Glenn bidirecional. Rev. Bras. Cir.
Cardiovasc., 7(3):194-200, 1992.

como de alto risco, para correção total, optando-se, entāo, pelo procedimento paliativo como preparação para a correção definitiva funcional.

Emtodos os pacientes submetidos a anastomoses de Glenn bidirecional com o auxílio da circulaçāo extracorpórea, a veia cava superior foi canulada na junção com a veia inominada. Mobilização da veia cava superior e ligadura da veia ázigos, quando indicada, foi realizada em todos os casos. Cuidado especial era tomado para nảo traumatizar o nó sinusal. A veia cava superior era seccionada na junção com o átrio direito, suturando-se a porção proximal e anastomosando-se a porção distal na artéria pulmonar com extremo cuidado para nāo distorcer a orientaçāo da veia cava e năo comprometer a drenagem venosa para os pulmōes.

\section{Resultados}

Nāo tivemos óbitos no período hospitalar ( 30 dias). O único óbito na série foi com 2 meses de pósoperatório, devido a infecção pulmonar, no primeiro paciente operado. Tratava-se de uma menina de 5 anos e 6 meses, portadora de ventrículo único tipo direito, com atresia mitral, veia cava superior esquerda persistente e bandagem do tronco pulmonar, atriosseptostomia com cirurgia realizada em 1985. Foi submetida a operação de Glenn bidirecional para a direita e esquerda e ligadura do tronco pulmonar. A saturação arterial inicial que era de $75,5 \%$ passou a $95 \%$ após a operaçāo. Evoluiu com hipersecreçāo pulmonar, hipoxia e entubaçāo prolongada. Foi reoperada em 28.9.90 (9 dias após a operaçāo de Glenn) para retirada da ligadura do tronco pulmonar. A saturaçāo pré era de $83 \%$ e, após retirada da ligadura, foi para $87 \%$. Evoluiu com melhora da saturação no pós-operatório, mas apresentou infecçāo pulmonar e atelectasia, que foram tratadas antibioticoterapia e corticóides, recebendo alta hospitalar em 23.10.90 (34 dia de pós-operatório). Reinternou dias após, com insuficiência cardiaca congestiva e derrame pleural, evoluindo para óbito com aproximadamente 2 meses de pós-operatório, devido a insuficiência respiratória e septicemia.

A pressão pulmonar variou entre $10 \mathrm{mmHg}$ a 20 $\mathrm{mmHg}$, com média de $14 \mathrm{mmHg}$. A saturação arterial média antes da correçāo era de $79 \%$ e, após, de $93 \%$.

No periodo de pós-operatório a extubação traqueal foi a mais precoce possivel e os pacientes eram mantidos em posiçăo semi-sentada. Sinais de insuficiência cardiaca congestiva estiveram presentes em $8 / 19$ casos, sendo tratados com digital e diuréticos.

No momento, está sendo criado um protocolo para avaliaçāo dos pacientes com ecocardiografia e ressonância eletromagnética, na qual poderemos observar e quantificar o fluxo sangüíneo para os pulmōes direito e esquerdo.

\section{COMENTÁRIOS}

Determinadas cardiopatias congênitas cianogênicas (atresia tricúspide, ventrículo único etc) necessita correção cirúrgica considerada paliativa definitiva, pela técnica de FONTAN et alii ${ }^{6,8,9} \mathrm{ou}$ sinais variantes ${ }^{15}$

Muitos pacientes não atingiriam a idade estabelecida para correçāo definitiva devido ao baixo fluxo pulmonar, necessitando, portanto, cirurgia paliativa para aumentar o fluxo pulmonar. Nestas condiçōes, a questão seria: qual é o melhor shunt preparatório, visando à correçāo definitiva. As opçōes cirúrgicas seriam o shunt sistêmico-pulmonar convencional, a anastomose de Glenn convencional, a operação de Gleen bidirecional e a técnica cirúrgica proposta pelo grupo da Mayo Clinic ${ }^{14}$, que está associada a alta mortalidade.

As vantagens da anastomose de Glenn sobre o shunt sistêmico-pulmonar convencional sāo as seguintes: reduz o trabalho para o ventrículo sistêmico, reduz o fluxo venoso para o átrio direito, shunt sangüíneo venoso em relaçāo à mistura artério-venosa e, finalmente, ausência da doença obstrutiva vascular pulmonar. Em resumo, a anastomose de Glenn convencional aumenta o fluxo efetivo pulmonar sem aumentar o trabalho cardíaco 2,16, 24.

A anastomose de Glenn bidirecional (shunt cavo-pulmonar bidirecional) apresenta vantagens sobre a técnica convencional (shunt unilateral): a continuidade entre as artérias pulmonares direita e esquerda está preservada, o fluxo sangüíneo pulmonar bilateral é obtido e a possibilidade de um subseqüente procedimento estará viável.

Os pacientes que não preencham os requisitos para a cirurgia de Fontan, ou que seriam considerados pacientes de alto risco para a cirurgia definitiva, devido à dimensão algo reduzida das artérias pulmonares, detectada durante o procedimento cirúrgico, poderāo ser tratados com a anastomose cavo-pulmonar bidirecional, aguardando a evoluçăo para o tatamento definitivo paliativo. LARBERTI et alii ${ }^{17}$ mostraram que de seus 17 pacientes tratados com shunt cavo-pulmonar bidirecional, 16 tiveram alta e $4(25 \%)$ casos em um período que variou de 24 a 39 meses de pós-operatório, foram submetidos a cirurgia de Fontan ( 3 casos) ou Björk ( 1 caso), com 1 óbito no $2^{\circ}$ ano de pós-operatório da cirurgia definitiva, devido a malformaçäo artéria-venosa pulmonar.

Três dos nossos pacientes tinham idade inferior a 1 ano (5 meses a 1 ano) e apresentaram evoluçāo 
CHACCUR, P.; DINKHUYSEN, J. J.; ABDULMASSIH NETO, C.; ARNONI, A. S.; SILVA,M. V. D.; BOSÍSIO, I. J.; FONTES, V. F.; CAPPELLARI, M.; PAULISTA, P. P.; JATENE, A. D.; SOUZA, L. C. B. - Operação de Glenn bidirecional. Rev. Bras. Cir. Cardiovasc., $7(3): 194-200,1992$.

pós-operatória semelhante ao grupo restante, com insuficiência cardíaca tratada com digitale diuréticos. $O$ paciente de 5 meses de idade apresentava diagnóstico de ventrículo único tipo direito, com atresia mitral a estenose pulmonar; foi submetido à operaçāo de Blalock-Hanlon, com melhora da saturaçāo de $68 \%$ para $88 \%$, mantendo hipoxemia na UTI, sendo indicada operaçāo de Glenn na manhã seguinte. Evoluiu sem intercorrências na UTI e no quarto apresentou insuficiência cardíaca discreta, tratada com digital e diuréticos. Fez ecocardiograma no $10^{\circ}$ dia de pós-operatório, que mostrou a anastomose cavopulmonar patente.

A operaçāo de Glenn bidirecional poderá ser realizada com ou sem auxílio da circulaçăo extracorpórea, na dependência do suprimento do fluxo pulmonar. Por exemplo, para os casos de BlalockTaussig à direita, seria mandatório o uso da CEC para a substituição da anastomose de Blalock pelo shunt cavo-pulmonar; nas situaçōes em que procedimentos intracardiacos sejam necessários e, finalmente, nos casos de dificuldades técnicas para efetuar a anastomose cavo-pulmonar. Os demais casos foram sempre tratados com shuntentre a veia inominada e o átrio direito, tomando-se o extremo cuidado ao se instalar o shunt para evitar embolia gasosa. A presença de veia cava inferior drenando na cava superior pela veia ázigos também poderá ser tratada com shuntextracardíaco, e a anastomose de Glenn bidirecional passará a ter um papel de cirurgia paliativa definitiva, uma vez que, somente as veias supra-hepáticas estariam drenando no átrio direito.

A presença da veia cava superior esquerda permite a realização de outra anastomose para a esquerda, diversificando, portanto, a drenagem venosa cefálica para a circulaçāo pulmonar. Nesta série, esta técnica cirúrgica foi empregada em $4(20 \%)$ pacientes.

Em conclusāo, podemos dizer que a ACP-bi (Glenn bidirecional) é uma ótima alternativa para aumentar o fluxo pulmonar em determinadas cardiopatias congênitas cianogênicas, apresentando índice de mortalidade muito baixo ou nulo. Em algumas situaçōes, poderá ser considerada cirurgia paliativa definitiva, na presença de veia cava inferior drenado na veia cava superior via ázigos, restando somente o sistema supra-hepático para drenar em átrio direito. Quanto à preparaçāo e à reduçāo de mortalidade durante a correçāo pela técnica de Fontan, ainda nāo temos essas respostas, embora alguns trabalhos já comecem a mostrar que alguns desses pacientes estão sendo submetidos à $2^{\mathrm{a}}$ fase do tratamento cirúrgico, com bons resultados.

CHACCUR, P.; DINKHUYSEN, J. J.; ABDULMASSIH NETO, C.; ARNONI, A. S.; SILVA, M. V. D.; BOSÍDIO, I. J.; FONTES, V. F.; CAPPELLARI, M.; PAULISTA, P. P.; JATENE, A. D.; SOUZA, L. C. B. - Bidirectional Glenn anastomosis. Rev. Bras. Cir. Cardiovac., 7(3):194-200, 1992.

ABSTRACT: The bidirectional Glenn anastomosis has bee used as an effective mean of palliating complex heart defects, in those patients that doesn't meet the necessary criteria for prosecution of an atriopulmonary anastomosis. The surgery consists on anastomosing the superior caval vein (end to side anastomoses), in a way that the venous blood is distributed to both the right and left lungs. Between January and February, 1992, 20 patients have undergone the bidirectional anastomosis at the Instituto Dante Pazzanese de Cardiologia. Eight patients were female. Their ages ranged from 5 months to 8 years (mean age 37.7 months) and their weights ranged from 6.3 to $18.8 \mathrm{~kg}$ (means $12.4 \mathrm{~kg}$ ). The surgical indication was considered primary in 10 cases. There were 8 patients with tricuspid atresia and 3 double inlet ventricles with highly diminished pulmonary blood flow considered not ideal candidates to a complete rapair. The other patients were considered secundary surgical indication. They had previous palliative operations such as systemic pulmonary shunts; pulmonary banding and atrialseptectomy ( 6 with tricuspid atresia, 3 double inlet ventricle with pulmonary stenosis or banding and 1 double intlet right ventricle with superior inferior ventricle). The surgery was performed with direction of the caval vein to right atrium in 12 cases and by means of cardiopulmonary bypass in 8 . During the operation, the patients were monitored with a pulsatile oximeter. The mean preoperative arterial oxygen saturation was $75.5 \%$ (range $71 \%$ to $86 \%$ ) and postoperative $95 \%$ (range $91 \%$ to $98 \%$ ). No hospital death occurred. There was one late death caused by pulmonary infection two months after the surgery. We believe that the bidirectional Glenn anastomosis has its place as a first stage to a complete correction, because it doesn't increase cardiac work and pulmonary vascular resistence and is not associated with pulmonary arterial and anastomotic distortions as Blalock-Taussig does.

DESCRIPTORS: Glenn bidirectional, surgery of. 
CHACCUR, P.; DINKHUYSEN, J. J.; ABDULMASSIH NETO, C.; ARNONI, A. S.; SILVA,M. V. D.; BOSÍSIO, I. J.; FONTES, V. F. CAPPELLARI, M.; PAULISTA, P. P.; JATENE, A. D.; SOUZA, L. C. B. - Operaçäo de Glenn bidirecional. Rev. Bras. Cir.
Cardiovasc., 7(3):194-200, 1992.

\section{REFERÊNCIAS BIBLIOGRÁFICAS}

1 ABRAMS, L. - Superior vena cava-pulmonary artery anastomosis. Ann. Thorac. Surg., 37: 9-11, 1984

2 ACHTEL, R. A.; KAPLAN, S.; BENZING, G.; HELMSWORTH, J. A. - Superior vena cava-right pulmonary artery anstomosis: long-term results. Ann. Thorac. Surg., 8: 511-519, 1969 .

3 BAKULJEV, A.; KOLESNIKOV, S. A. - Anastomosis of superior vena cava and pulmonary artery in the surgical treatment of certain congenital of the heart. J. Thorac. Surg., 37:693, 1959.

4 CALABRESE, C. T.; CARRINGTON, C. B.; HARLEY, R. A.; ROJAS, R. H.; GLENN, W. W. L. - Circulatory bypass of the right side of the heart. J. Surg. Res. 8: $593-600,1968$,

5 CARLON, C. A.; MONDINI, P. G.; de MARCHI, R. Surgical treatment of some cardiovascular disease. J. Int. Coll. Surg., 16: 1-10, 1951.

6 De BRUX, J. L.; ZANNINI, L.; BINET, J. P. - Tricuspid atresia: results of treatment in 115 children. J. Thorac. Cardiovasc. Surg., 85: 440-446, 1982.

7 DeLEVAL, M. R.; BULL, C.; KILNER, P. - Total cavopulmonary connection: a logical alternative to atriopulmonary connection for complex Fontan operations: experimental studies and early clinical experience. Ann. Thorac. Surg., 46:210-215, 1988.

8 FONTAN, F. \& BAUDET, E. - Surgical repair of tricuspid atresia. Thorax, 26: 240-248, 1971.

9 FONTAN, F.; De VILLE, C.; QUAEGEBEUR, J.; OTTEM KAMP, J.; SOURDILLE, N.; CHOUSSAT, A.; BROM, G. A. - Repair of tricuspid atresia in 100 patients. J. Thorac. Cardiovasc. Surg., 85: 647-660, 1983.

10 GLENN, W. W. L. - Circulatory bypass of the right side of the heart. IV. Shunt between superior vena cava and distal right pulmonary artery: report of clinical application. N. Engl. J. Med., 259: 117-120, 1958.

11 GRAHAM, A. J.; RICKETTS, H. J.; FENN, J. E.; LARSEN, P. B.; MUZAFFER, C.; GLENN, W. W. L. - Further experiments on long term survivors after circulatory bypass of the right side of the heart. Surg. Obstet. Gynecol., 119: 302-310, 1964.

12 HALLER, J. A.; ADKINS, J. C.; WORTHINGTON, M.; RAUENHORST, J. - Experimental studies on permanent bypass of the right heart. Surgery, 59: 1128-1132, 1966.

13 KAWASHIMA, Y.; KITAMURA, S.; MATSUDA, $H_{\text {.; }}$ SHIMAZAKI, Y.; NAKANO, S.; HIROSE, H. - Total cavopulmonary shunt operation in complex cardiac anomalies. J. Thorac. Cardiovasc. Surg., 87: 7481-87, 1984.
14 KING, R. M.; PUGA, F. J.; DANIELSON, G. K. - Extende indications for the modified Fontan procedure in patients with anomalous systemic and pulmonary venous return. In. DOYLE, E. F.; ENGLE, M. A.; GERSONY, W. M.; RASHKIND, W. J.; TULNER, N. S. (eds.) Proceedings of the Second World Congress of Pediatric Cardiology. New York, Springer-Verlag. 1985.

15 KREUTZER, F. A. - Atriopulmonary anastomosis. J. Thorac. Cardiovasc. Surg., 83: 427-436, 1982.

16 LAKS, H.; MUDD, G. J.; STANDEVEN, J. W.; WILLIAM, V. L. - Long-term effect of the superior vena cavapulmonary artery anastomosis on pulmonary blood flow. J. Thorac. Cardiovasc. Surg., 74: 253-260, 1977.

17 LAMBERTI, J. J.; SPICER, R. L.; WELDMAN, J. D.; GREHL, T. M.; THOMSON, D.; GIORGE, L.; KIRKPATRIC, S. E.; MATHEWSON, J. W. - The bidirectional cavopulmonary shunt. J. Thorac. Cardiovasc. Surg., 100: 22-30, 1990.

18 MAZZERA, E.; CORNO, A.; PICARDO, S.; DIDONATO, R.; MARINO, B.; COSTA, D.; MARCELLETTI, C. Bidirectional cavopulmonary shunts: clinical applications as staged or definitive palliation. Ann. Thorac. Surg., 47: 415-420, 1989.

19 MESHALKIN, E. N. - Anastomosis of the superior vena cava with the pulmonary artery in patients with congenital heart disease with blood flow insufciency in the lesser circulation. Experiment. Eksp. Khirurgie, 1: 3, 1956

NULAND, S. G.; GLENN, W. W. L.; GUILFOIL, P. H. Circulatory bypass of the right heart: III. Some observations on the long-term survivors. Surgery, 43: $184-201,1958$.

21 PATINO, J. F.; GLENN, W. W. L.; GUILFOIL. P. H. Circulatory bypass of the right heart: II. Further observatiuons on vena caval-pulmonary artery shunts. Surg. Forum, 6: 189-193, 1955.

22 ROBCSEK, F.; TEMESVARI, A.; KADAR, R. L. - A new method for the treatment of congenital heart disease associated with impaired pulmonary circulation. Acta. Med. Scand., 154: 154-161, 1956.

23 SHUMACKER, H. B.; (discussion of Hurwitt, E.; YOUNG, D.; ECHER, D.) - Rationale of anastomosis of right auricular appendage to pulmonary artery in the treatment of tricuspid atresia. J. Thorac. Surg., 30: 503-512, 1955.

24 YOUNG, W. G.; SEALY, W. C.; HOUCK, W. S. - Superior vena cava-right pulmonary anastomosis in cyanotic heart disease. Ann. Surg., 157: 894-901, 1963. 
CHACCUR, P.; DINKHUYSEN, J. J.; ABDULMASSIH NETO, C.; ARNONI, A. S.; SILVA,M. V. D.; BOSÍSIO, I. J.; FONTES, V. F.; CAPPELLARI, M.; PAULISTA, P. P.; JATENE, A. D.; SOUZA, L. C. B. - Operação de Glenn bidirecional. Rev. Bras. Cir. Cardiovasc., 7(3):194-200, 1992.

\section{Discussāo}

\section{DR. HAROLDO OLIVEIRA Belém, PA}

Cumprimento Dr. Chaccur e colaboradores, pela oportunidade de reapresentaçāo desta técnica, que nos parece muito útil nas cirurgias de exclusão do coração direito, visando ao aumento do fluxo pulmonar nas cardiopatias complexas. Como fundamento para o sucesso desta operaçāo, a baixa resistência pulmonar determina a facilidade de fluxo imediato pelo leito vascular pulmonar, após a derivação. Entretanto, a recuperaçāo destes pacientes é arrastada pelas contínuas efusōes dos territórios venosos, a nível de peritôneo, pleuras, mesmo pericárdio, obrigando tratamento médico cuidadoso e monitoração freqüente nas primeiras semanas após a operaçāo, existindo Serviços que rotineiramente deixam drenagem torácica bilateral por tempo prolongado para evitar coleçōes. Visto que, em sua casuística, alguns casos apresentaram variaçōes anatômicas no sistema de drenagem venosa, que, com alguma freqüência, se associam ao defeito congênito do coração. Como em sua casuística, que reflete a excelência do seu trabalho, existem casos em que foi feita a ligadura da veia ázigos, junto à cava superior direita, gostaria de saber como foi feita a avaliação do sistema de retorno venoso ao coraçāo, de forma a indicar a ligadura da veia ázigos, sem que levasse a uma maior estase no leito do sistema sistema nervoso, conseqüentemente, propiciando complicaçōes no pós-operatório. Parabenizo o Dr. Chaccur e colaboradores pela excelência do trabalho e a oportunidade da apresentação. Agradeço à Comissāo Organizadora o privilégio da indicaçāo para comentar este trabalho.

\section{DR. CHACCUR \\ (Encerrando)}

Agradeço os comentários ao Dr. Haroldo Oliveira e gostaria de concluir dizendo que tem sido pouco freqüente, em nossa casuística, os casos de derrames pleurais ou peritoniais, nestes pacientes submetidos a cirrugia de Glenn bidirecional, como primeira etapa no tratamento cirúrgico das derivaçōes atriopulmonares. Concordamos como Dr. Haroldo em que existem variaçōes anatômicas nas drenagens venosas sistêmicas, sendo e mais freqüente a veia cava superior esquerda persistente drenando em seio coronário. Para estes casos, uma outra anastomose de Glenn bidirecional deverá ser realizada. Para as anomalias de drenagem da veia cava inferior, principalmente quando estas drenam via sistema ázigos, estarem impossibilitados de ligá-las, para confecçāo da anastomose de Glenn. A avaliaçāo pré-operatória por ecocardiograma e o estudo hemodinâmico normalmente nos dāo estas informaçōes. O próprio as pecto cirúrgico de uma veia ázigo bastante aumentada também poderá chamar atençāo para anomalias de drenagem venosa, impossibilitando-nos para ligadura das mesmas. Agradeço, novamente, ao Dr. Haroldo, pelos comentários, e à Comissāo Organizadora deste Congresso pela escolha deste trabalho para apresentação em plenário. Obrigado. 